\title{
REDES DE PETRI HÍBRIDAS DIFERENCIAIS: APLICAÇÃO NA MODELAGEM E NO GERENCIAMENTO DINÂMICO DE ENERGIA DE REDES DE SENSORES SEM FIO
}

\author{
J. R. B. Sousa* \\ renato@cefet-ce.br \\ A. M. N. Lima ${ }^{\ddagger}$ \\ amnlima@dee.ufcg.edu.br
}

\author{
P. S. Sausen ${ }^{\dagger}$ \\ sausen@unijui.tche.br \\ A. Perkusich $\ddagger$ \\ perkusic@dee.ufcg.edu.br
}

${ }^{*}$ CEFETCE - Centro Federal de Educação Tecnológica do Ceará

Área da Indústria - Curso de Mecatrônica

Av. 13 de Maio, 2081 - Benfica - 60.040-531

Fortaleza, Ceará, Brasil

†UNIJUÍ - Universidade Regional do Noroeste do Estado do RS

Departamento de Tecnologia

Rua São Francisco, 501 - São Geraldo - 98.700-000

Ijuí, Rio Grande do Sul, Brasil

${ }^{\ddagger}$ UFCG - Universidade Federal de Campina Grande

Departamento de Engenharia Elétrica

Av. Aprigio Veloso, 882 - Bodocongó - 58.109-970

Campina Grande, Paraíba, Brasil

\section{ABSTRACT}

In this work a formalism named Differential Hybrid Petri Nets to model and simulate hybrid systems is presented. It is based on the main elements of the semantics of the hybrid automaton and the modeling power of Petri nets. Considering that Wireless Sensor Networks are hybrid systems, this formalism is applied to model a Dynamic Power Management technique based on battery Capacity (GDC). Therefore, when taken into account the interactions among the dynamics of these nets, more realistic results are obtained in

ARTIGO CONVIDADO:

Versão completa e revisada de artigo apresentado no SBAI-2005

Artigo submetido em 01/06/2006

1a. Revisão em 14/09/2006

2a. Revisão em 03/04/2007

Aceito sob recomendação do Editor Convidado

Prof. Osvaldo Ronald Saavedra Mendez the study of energy consumption. The proposed GDC technique, in function of the capacity of the battery, defines the turn off frequency of the sensor node to allow the recovery of battery capacity. Besides, the proposed GDC technique minimizes the turn off time of the sensor node, at same time that distributes this time along the cycles of work of the sensor node. The obtained results demonstrate that the proposed GDC technique prolongs the lifetime of the sensor node.

KEYWORDS: Hybrid Systems, Petri Nets, Wireless Sensor Networks, Dynamic Power Management.

\section{RESUMO}

Neste trabalho é apresentado um formalismo denominado Redes de Petri Híbridas Diferenciais para modelar e simular sistemas híbridos. Ele baseia-se nos principais elementos da 
semântica do autômato híbrido e no poder de modelagem das redes de Petri. Considerando que Redes de Sensores Sem Fio são sistemas híbridos, este formalismo é aplicado na modelagem de uma técnica de Gerenciamento Dinâmico de energia que baseia-se na Capacidade da bateria (GDC). Portanto, ao levar em conta as interações entre as dinâmicas destas redes obtém-se resultados mais realistas no estudo do consumo de energia. A técnica GDC proposta, em função da capacidade da bateria, define a frequiência de desligamento do nó sensor para permitir a recuperação da capacidade da bateria. Além disto, ela minimiza o tempo de desligamento do nó sensor, ao mesmo tempo que distribui esse tempo ao longo dos ciclos de trabalho do mesmo. Os resultados obtidos mostram que a técnica GDC proposta prolonga o tempo de vida do nó sensor.

PALAVRAS-CHAVE: Sistemas Híbridos, Redes de Petri, Redes de Sensores Sem Fio, Gerenciamento Dinâmico de Energia.

\section{INTRODUÇÃO}

O grande avanço nas áreas de processadores de baixo consumo, dispositivos de sensoriamento, sistemas embarcados e comunicação sem fio tem estimulado o desenvolvimento e a utilização das Redes de Sensores Sem Fio (RSSFs) numa ampla faixa de aplicações. Estas redes são compostas de centenas ou milhares de dispositivos autônomos e compactos chamados nós sensores. As RSSFs são utilizadas, por exemplo, em aplicações militares e de segurança, na automação da manufatura, no monitoramento de estruturas de edifícios e de sistemas ambientais e também na agricultura de precisão (Vieira et al., 2003).

As RSSFs são sistemas nos quais duas dinâmicas, uma a eventos discretos e outra contínua, coexistem e interagem (Passos et al., 2005). Este tipo de comportamento caracteriza os sistemas denominados híbridos. Numa RSSF, a dinâmica a eventos discretos é representada pelo hardware e software dos nós sensores. Por outro lado, a dinâmica contínua é representada pelo comportamento dos parâmetros do sistema de energia que alimenta cada nó sensor e pelas variáveis ambientais monitoradas pela rede.

Nas últimas décadas os Sistemas Híbridos (SHs) têm recebido muita atenção. Eles estão presentes em muitas aplicações, tais como, controle de processos químicos, sistemas de gerenciamento de tráfego aéreo, robótica, redes de sensores, sistemas de fornecimento de energia e automóveis. Vários modelos têm sido propostos com o objetivo de definir um formalismo matemático que possibilite lidar com o seu comportamento dinâmico complexo.

De modo geral, a abordagem seguida pelos cientistas de com- putação é a de estender o modelo autômato de estado finito com a introdução de dinâmicas contínuas mais complexas. Pesquisadores da área de controle, por outro lado, tratam os SHs pela incorporação de comportamentos discretos dentro das descrições da dinâmica contínua, especificamente, equações diferenciais ordinárias (Dang, 2000). A Dinâmica contínua é tradicionalmente consideradas dentro do contexto das equações diferenciais, e a dinâmica a eventos discretos é geralmente modelada e analisada usando-se autômatos ou Redes de Petri (Murata, 1989), por exemplo.

A contribuição das Redes de Petri (RP) para a modelagem e análise de sistemas a eventos discretos é bem conhecida. Para modelar SHs, uma extensão de RPs é necessária para representar os componentes da dinâmica contínua. Neste artigo é apresentado um formalismo baseado em RPs que integra as vantagens dos formalismos apresentados em (LeBail et al., 1991), (Demongodin and Koussoulas, 1996), (Daubas et al., 1994), (Champagnat, 1998) e (Wu et al., 2002). Este formalismo é denominado Redes de Petri Híbridas Diferenciais (RPHDs), cujo propósito é modelar e simular SHs, baseando-se nos principais elementos da semântica do autômato híbrido e no poder de modelagem das RPs. A utilização das RPHDs no estudo de SHs possibilita modelar, explorando o poder de representação das RPs, as características da dinâmica a eventos discretos, ou seja, concorrência, sincronismo, conflito, exclusão mútua, relações de precedência, não determinismo e bloqueio.

Em muitas aplicações, RSSFs têm os seus nós colocados em áreas remotas de difícil acesso, o que não permite, facilmente, o acesso a esses elementos para manutenção. Uma vez que é impraticável recarregar a bateria desses nós, cada nó deve ser eficiente em energia. Portanto, energia é o principal recurso de um nó sensor, sendo a métrica primária para análise e projeto de RSSFs (Vieira et al., 2003). Para estudar o consumo de energia de uma RSSF de forma mais realista é necessário: (i) levar em conta as interações entre as dinâmicas inerentes a uma RSSF dentro do contexto de SHs, e (ii) utilizar um modelo preciso de bateria para a obter uma previsão correta do tempo de vida de um nó sensor. Assim, neste trabalho, utiliza-se uma abordagem de (Sousa et al., 2005) para modelar e analisar o consumo de energia de um nó sensor. Desta forma, o formalismo RPHD é utilizado para modelar uma técnica de gerenciamento dinâmico de energia para um nó sensor, com o objetivo de prolongar o tempo de vida do mesmo.

As seções restantes deste artigo são organizadas da seguinte maneira: os trabalhos correlatos na modelagem de SHs com RPs são apresentado na Seção 2. A seguir na Seção 3, as RPHDs são apresentadas. Na Seção 4, as RSSFs são apresentadas dentro do contexto de SHs. Além disso, é apresentada a proposta de uma técnica de gerenciamento dinâmico de ener- 
gia com o seu modelo RPHD. Um modelo RPHD para um nó sensor, que utiliza esta técnica de gerenciamento de energia é apresentado na Seção 5. Os resultados de simulação da técnica de gerenciamento proposta são apresentados na Seção 6. Finalmente, as conclusões são apresentadas na Seção 7.

\section{MODELANDO SISTEMAS HÍBRIDOS COM REDES DE PETRI}

Para modelar e analisar formalmente SHs, é necessário um modelo matemático que seja capaz de descrever suas dinâmicas e as interações que ocorrem entre estas. Portanto, é vantajoso e indispensável, poder representar ambas as dinâmicas de um SH num mesmo contexto (Demongodin and Koussoulas, 1996). A classe de SHs considerada neste trabalho caracteriza-se por uma dinâmica a eventos discretos com maior complexidade do que a dinâmica contínua. Desta forma, foi escolhido o domínio dos sistemas a eventos discretos como estrutura base de modelagem.

As abordagens que especificam uma solução de modelagem a partir da integração de um formalismo a eventos discretos com um formalismo contínuo apresentam maior flexibilidade e maior poder de modelagem. Entre as abordagens deste grupo, neste trabalho considerou-se em especial aquelas onde o formalismo a eventos discretos baseia-se em RPs. Esta escolha foi realizada considerando-se as já bem conhecidas características das RPs, tais como a possibilidade de representar a sincronização de processos, concorrência, causalidade, compartilhamento de recursos, presença de conflitos, entre outras.

Modelar SHs com RPs apresenta o problema de como representar a dinâmica contínua dentro da estrutura de uma RP. A necessidade de uma extensão para resolver este problema motivou a criação de vários formalismos. Um passo no sentido de incorporar dinâmicas contínuas a um formalismo a eventos discretos foi realizado ao se definir as RPs Contínuas (RPCs) (David and Alla, 1987). A combinação de RPs contínuas com RPs temporizadas deu origem ao conceito de RPs Híbridas (RPHs). Porém, esses formalismos não permitem a representação de variáveis contínuas negativas e tornam difícil a modelagem de sistemas com ordem maior do que um.

Vários formalismos originaram-se a partir das RPHs (LeBail et al., 1991). As RPs Globais são propostas em (Rezai et al., 1995), nestas a concepção de marcação de lugar é ampliada para vetores e a função definida para os arcos é matricial, o que resulta num modelo mais compacto. Drath, apresenta uma classe de RP Híbrida estendida para a modelagem de SHs (Drath, 1998). A modelagem de sistemas complexos é realizada através da aplicação dos conceitos de orientação a objetos nas RPs Híbridas, obtendo-se assim as RPs Híbridas Objeto. Nestas, a capacidade para modelar SHs é estendida através da inclusão de um novo tipo de arco denominado arco teste, e através de um novo conceito para as velocidades de disparo das transições contínuas.

A partir dos conceitos das RPs contínuas com velocidade máxima de disparo dependente do tempo (Dubois et al., 1994), foram definidas as RPs Diferenciais Temporizadas (Demongodin and Koussoulas, 1996). Este formalismo permite a modelagem de variáveis contínuas negativas, entretanto, para uma dinâmica contínua de ordem elevada, a estrutura deste formalismo torna-se complexa.

Uma abordagem que combina RPs e equações diferenciais, foi introduzida em (Daubas et al., 1994). Nesta, nenhuma restrição é imposta às equações da dinâmica contínua, e o seu cálculo é um processo implícito a cada lugar da rede. Seguindo esta mesma forma de tratar a interação entre as dinâmicas de um SH, em (Champagnat, 1998), foi introduzido o formalismo RPs Predicado/Transição Diferencial. Este formalismo apresenta algumas semelhanças com o autômato híbrido (Dang, 2000). O autômato híbrido é uma ferramenta poderosa para analisar SHs devido os seus métodos de análise. Entretanto, a modelagem baseada em autômato híbrido nem sempre é uma tarefa fácil, já que a semântica associada a este formalismo baseia-se na definição prévia dos estados discretos do modelo. Além disso, a complexidade do modelo cresce consideravelmente com a ordem do sistema.

Aproveitando as vantagens das RPs Diferenciais Temporizadas e RPs Globais, foram propostas as RPs Diferenciais Generalizadas (RPDGs). Esta extensão permite que métodos de integração numérica (e.g., Euler e Runge-Kutta) sejam utilizados para simular dinâmicas contínuas (Wu et al., 2002). Embora este formalismo utilize o conceito de marcação apresentado em (Rezai et al., 1995), ele apresenta o inconveniente de procurar representar o sistema de equações diferenciais que representa a dinâmica contínua do $\mathrm{SH}$, tal como em (Demongodin and Koussoulas, 1996).

Villani, apresenta um formalismo denominado Rede de Petri Predicado-Transição Diferencial Orientada a Objetos, para modelar e analisar sistemas supervisórios híbridos (Villani, 2004). Neste formalismo, os conceitos de orientação a objetos são incorporados ao formalismo de (Champagnat, 1998). A principal vantagem desta incorporação é a adoção de uma estrutura modular, que permite o enfoque em apenas uma parte do sistema de cada vez, facilitando a modelagem de sistemas complexos e de grande porte.

Os formalismos propostos em (LeBail et al., 1991; Demongodin and Koussoulas, 1996; Giua and Usai, 1998; Drath, 1998; Wu et al., 2002), modelam o processo de integração numérica da equação diferencial ou do sistema de equações diferenciais, que representa a dinâmica contínua do SH estudado. Esta abordagem faz com que sistemas com ordem 
elevada tenham um modelo híbrido com uma estrutura muito complexa. Drath lida com este problema através da aplicação dos conceitos de orientação a objetos (Drath, 1998).

Outro trabalhos: (Daubas et al., 1994), (Champagnat, 1998) e (Villani, 2004), procuram reduzir esta complexidade considerando o cálculo da evolução contínua como um processo implícito da semântica do modelo a eventos discretos, tal como ocorre no autômato híbrido. Desta forma, o modelo $\mathrm{RP}$ apresenta uma estrutura reduzida.

O formalismo apresentado neste trabalho baseia-se na abordagem seguida por (Daubas et al., 1994) e (Champagnat, 1998). Além disto, ele procura explicitar e enfatizar as interações que ocorrem entre as dinâmicas de um SH. Este formalismo é denominado Redes de Petri Híbridas Diferenciais (RPHDs) e é apresentado na próxima seção.

\section{REDES DE PETRI HÍBRIDAS DIFEREN- CIAIS}

Uma Rede de Petri Híbrida Diferencial (RPHD) é composta de dois tipos de lugares e dois tipos de transições: lugares e transições discretos, lugares e transições diferenciais. Um lugar diferencial é representado por três círculos concêntricos e uma transição diferencial por dois retângulos concêntricos. Um lugar e uma transição discretos são representados por um círculo e uma barra, respectivamente. O diagrama da Figura 1 ilustra os tipos de lugares e transições de uma RPHD.

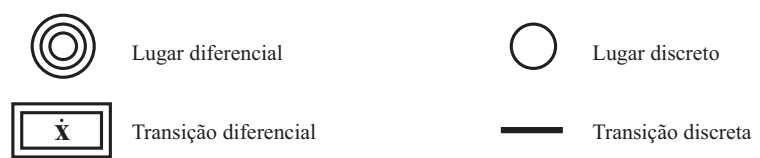

Figura 1: Lugares e transições de uma RPHD.

Uma RPHD possui três tipos de arcos. Dois tipos denominados arco normal e arco inibidor, enquanto que o terceiro é denominado arco teste. O arco normal e o arco inibidor podem ser usados para conectar os elementos discretos da rede, com exceção para o arco que conecta um lugar diferencial e uma transição discreta, onde é usado o arco teste. Na Figura 2 são mostrados os tipos de arcos de uma RPHD.

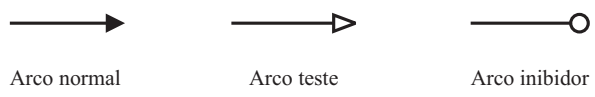

Figura 2: Tipos de arcos de uma RPHD.

Definição 1 Uma Rede de Petri Híbrida Diferencial é definida como $R P H D=\left(\mathbf{X}, P, T, f, A_{N}, A_{I}, A_{T}, \operatorname{Pre}\left(P_{i}, T_{j}\right)\right.$, $\left.\operatorname{Post}\left(P_{i}, T_{j}\right), \mathcal{T}, H_{P_{i}, T_{j}}, J_{T_{j}, P_{i}}, M_{0}(t)\right)$, onde
- $\mathbf{X}=\left[x_{1}, x_{2}, \ldots, x_{l}\right]^{T}$, é o vetor de estado contínuo, onde $\mathbf{X} \subset \mathbb{R}^{l}$ e $l$ é a dimensão do sistema;

- $\mathbf{X}_{0}=\left[x_{1}(0), x_{2}(0), \ldots, x_{l}(0)\right]^{T}$, é o vetor de estado contínuo inicial do sistema;

- $P=\left\{P_{1}, P_{2}, \ldots, P_{n}\right\}$, é um conjunto finito e não vazio de lugares. Onde $n$ é a cardinalidade de $P$;

- $T=\left\{T_{1}, T_{2}, \ldots, T_{m}\right\}$, é um conjunto finito e não vazio de transições. Onde $m$ é a cardinalidade de $T$;

$$
\text { - } P \cap T=\varnothing \text { e } P \cup T \neq \varnothing ;
$$

- $f: P \cup T \rightarrow\{D, D F\}$, é chamada função diferencial, onde $D$ significa discreto e $D F$ significa diferencial, indica se um lugar $P_{i}$ (uma transição $T_{j}$ ) é um lugar (transição) discreto ou um lugar (transição) diferencial. As notações $\left\{P_{D}, T_{D}\right\}$ e $\left\{P_{D F}, T_{D F}\right\}$, também são usadas para representar os conjuntos de elementos discretos e diferenciais, respectivamente;

- $P=P_{D} \cup P_{D F}$ e $T=T_{D} \cup T_{D F}$;

- O conjunto de lugares discretos $\left(f\left(P_{i}\right)=D\right)$ modela o estado discreto de um $\mathrm{SH}$;

- Uma transição discreta $\left(f\left(T_{j}\right)=D\right)$ modela a ocorrência de um evento discreto num $\mathrm{SH}$;

- Um lugar diferencial $\left(f\left(P_{i}\right)=D F\right)$ modela o estado contínuo de um SH;

- Uma transição diferencial $\left(f\left(T_{j}\right)=D F\right)$ modela o cálculo da evolução contínua de um SH;

- $A_{N} \subseteq\left(\left(P_{D} \times T_{D}\right) \cup\left(T_{D} \times P_{D}\right)\right) \cup\left(\left(P_{D} \times T_{D F}\right) \cup\right.$ $\left.\left(T_{D F} \times P_{D}\right)\right)$, é um conjunto de arcos normais;

- $A_{I} \subseteq\left(P_{D} \times T_{D}\right)$, é um conjunto de arcos inibidores;

- $A_{T} \subseteq\left(P_{D F} \times T_{D}\right) \cup\left(T_{D} \times P_{D F}\right)$, é um conjunto de arcos teste;

- $\operatorname{Pre}\left(P_{i}, T_{j}\right): P_{D} \times T_{D} \rightarrow \mathbb{N}$, é uma função que define os arcos normais de um lugar $P_{i}$ para uma transição $T_{j}$.

- $\operatorname{Post}\left(P_{i}, T_{j}\right): P_{D} \times T_{D} \rightarrow \mathbb{N}$, é uma função que define os arcos normais de uma transição $T_{j}$ para um lugar $P_{i}$.

- $\mathcal{T}$ é um mapa de temporização para as transições discretas. Diferentemente do que propõe (Wu et al., 2002), no presente caso não é necessário definir um atraso para as transições diferenciais;

- $H_{P_{i}, T_{j}}: P_{D F} \times T_{D} \rightarrow \mathbf{X}$, é uma função de habilitação ${ }^{1}$ associada ao arco teste que conecta um lugar diferencial de entrada $P_{i}$ à transição discreta $T_{j}$. A habilitação de $T_{j}$ é definida de acordo os valores das variáveis de estado $x_{k}$ associadas com a marcação do lugar $P_{i}$;

\footnotetext{
${ }^{1}$ Em (Champagnat, 1998), estas funções são associadas à transições discretas.
} 
- $J_{T_{j}, P_{i}}: T_{D} \times P_{D F} \rightarrow \mathbf{X}$, é uma função de junção ${ }^{1}$ associada ao arco teste que conecta uma transição discreta $T_{j}$ a um lugar diferencial de saída $P_{i} . J_{T_{j}, P_{i}}$ define os novos valores das variáveis de estado $x_{k}$ associadas com a marcação do lugar diferencial $P_{i}$ após o disparo de $T_{j}$. Para os casos em que $J_{T_{j}, P_{i}}$ não é definida, $J_{T_{j}, P_{i}}$ é a função identidade;

- $M_{0}(t): P \rightarrow \mathbb{N}$ ou $\mathbb{R}^{l}$, representa a marcação inicial da rede em $t=0$. Para um lugar específico $P_{i}$, a marcação inicial é representada por $m_{0}\left(P_{i}\right)(t)$.

A evolução no tempo de $\mathbf{X}$ é dada por $\dot{\mathbf{X}}=\mathrm{f}(\mathbf{X}, t)$. Para cada transição diferencial, é atribuída uma condição de fluxo $\dot{\mathbf{X}}$, a qual é utilizada no cálculo da respectiva evolução contínua.

\subsection{Marcação de uma RPHD}

A marcação de uma RPHD é representada por $m\left(P_{i}\right)(t)$, ou seja, a marcação de um lugar $P_{i}$ no tempo $t$ depende do tipo de lugar.

- Para um lugar discreto, a marcação é um inteiro não negativo, ou seja, se $f\left(P_{i}\right)=D$, então $m\left(P_{i}\right)(t) \in \mathbb{N}$.

- Para um lugar diferencial, a marcação é um vetor real, ou seja, se $f\left(P_{i}\right)=D F$, então $m\left(P_{i}\right)(t)=\mathbf{X}$ e $m_{0}\left(P_{i}\right)(t)=\mathbf{X}_{0}$. A marcação de um lugar diferencial representa o vetor de estado contínuo do SH.

\subsection{Mapa de Temporização}

Para uma transição discreta: se $f\left(T_{j}\right)=D$, então $\mathcal{T}\left(T_{j}\right)=$ $d_{j} \in \mathbb{R}^{\geq 0}$, onde $d_{j}$ é o tempo de atraso associado a transição $T_{j}$. Se $d_{j}=0$, então a transição $T_{j}$ executará o seu disparo imediatamente após ser habilitada.

\subsection{Condições Particulares para a estru- tura de uma RPHD}

Sejam ${ }^{\bullet} T_{j}\left(\bullet_{i}\right)$ e $T_{j}^{\bullet}\left(P_{i}^{\bullet}\right)$ os conjuntos de predecessores e sucessores da transição $T_{j}$ (do lugar $P_{i}$ ), respectivamente.

1. Se um arco normal conecta um lugar discreto a uma transição diferencial, deve existir outro arco normal conectando esta transição diferencial ao mesmo lugar discreto. Além disso, esses arcos normais devem ter o mesmo peso. Formalmente, se $f\left(P_{i}\right)=D$ e $f\left(T_{j}\right)=D F$ tal que $P_{i} \in{ }^{\bullet} T_{j}$ (resp. $P_{i} \in T_{j}^{\bullet}$ ), então $P_{i} \in T_{j}^{\bullet}$ (respectivamente $\left.P_{i} \in{ }^{\bullet} T_{j}\right)$ e $\operatorname{Pre}\left(P_{i}, T_{j}\right)=$ $\operatorname{Post}\left(P_{i}, T_{j}\right)$. Esta condição considera que a marcação de todos os lugares discretos é um inteiro para qualquer evolução da rede. Esta condição pode ser observada no modelo RPHD mostrado na Figura 3(a). Neste caso, os dois arcos conectando o lugar discreto $P_{1}$ e a transição diferencial $T_{2}$ garantem a integridade da marcação de $P_{1}$.

2. Um arco normal pode conectar uma transição diferencial a um lugar diferencial, mas o contrário, não é permitido. Formalmente, se $f\left(P_{i}\right)=D F$ e $f\left(T_{j}\right)=D F$, então ${ }^{\bullet} T_{j} \cap P_{i}=\emptyset$. Esta condição, contudo, não limita o poder de modelagem porque: a) o estado de um lugar diferencial não influencia diretamente a habilitação/desabilitação de uma transição diferencial; b) a evolução da marcação do lugar $P_{i}$ é determinada pela dinâmica contínua associada à transição $T_{j}$ de entrada e não pelo sentido do arco normal que os conecta. Esta condição é ilustrada no modelo RPHD da Figura 3(a). Note que a habilitação de $T_{2}$ não depende da marcação de $P_{2}$, e que embora exista um arco direcionado de $T_{2}$ para $P_{2}$, isto não implica que a marcação contínua de $P_{2}$ deverá, obrigatoriamente, crescer. Para confirmar este fato, o leitor deve observar a evolução das variáveis de estado contínuo $x_{1}$ e $x_{2}$ na Figura 3(b).

\subsection{Regras de Evolução}

A evolução de uma RPHD é definida pelo disparo de transições. Para cada tipo de transição, as condições para habilitação e disparo são diferentes.

\subsubsection{Condições de Habilitação}

Definição 2 Uma transição $T_{j}$ está habilitada no tempo $t$ se e somente se:

1. Se $T_{j}$ é uma transição discreta, cada lugar discreto de entrada tem uma marcação pelo menos igual ao peso do arco normal correspondente. Para aqueles lugares discretos de entrada que conectam-se através de arcos inibidores, esses lugares têm uma marcação inferior ao peso dos arcos inibidores correspondentes. Além disso, para cada lugar diferencial de entrada, a respectiva função de habilitação $H_{P_{i}, T_{j}}$ tem predicado verdadeiro, ou seja, se $f\left(T_{j}\right)=D$, então $\forall P_{i} \in P_{D} \cap^{\bullet} T_{j}, m\left(P_{i}\right)(t) \geq$ $\operatorname{Pre}\left(P_{i}, T_{j}\right)$, e $\forall P_{i} \in P_{D F} \cap{ }^{\bullet} T_{j}, H_{P_{i}, T_{j}}=$ verdade;

2. Se $T_{j}$ é uma transição diferencial, cada lugar discreto de entrada tem uma marcação pelo menos igual ao peso do arco normal correspondente, ou seja, se $f\left(T_{j}\right)=D F$, então $\forall P_{i} \in P_{D} \cap^{\bullet} T_{j}, m\left(P_{i}\right)(t) \geq \operatorname{Pre}\left(P_{i}, T_{j}\right)$. Veja o modelo RPHD mostrado na Figura 3(a), onde o lugar discreto $P_{1}$ tem uma marcação suficiente para habilitar a transição diferencial $T_{2}$. 
Nota 1 Não é permitido atribuir simultaneamente à uma transição discreta $\left(T_{j} \in T_{D}\right)$ um tempo de atraso $d_{j}$ e uma função de habilitação $H_{P_{i}, T_{j}}$.

\subsubsection{Condições de Disparo}

Quando uma transição discreta dispara, um número de fichas igual ao peso do arco normal que liga cada lugar discreto de entrada à transição discreta é removido do respectivo lugar discreto de entrada, enquanto um número de fichas igual ao peso do arco normal que liga à transição cada lugar discreto de saída é adicionado ao respectivo lugar discreto de saída. Formalmente, se $T_{j}$ esté habilitada no tempo $t, f\left(T_{j}\right)=D$ e $\mathcal{T}\left(T_{j}\right)=d_{j}$, então $T_{j}$ dispara no tempo $t+d_{j}, \forall P_{i} \in$ - $T_{j}, m\left(P_{i}\right)\left(t+d_{j}\right)=m\left(P_{i}\right)(t)-\operatorname{Pre}\left(P_{i}, T_{j}\right)$ e $\forall P_{i} \in T_{j}^{\bullet}$, $m\left(P_{i}\right)\left(t+d_{j}\right)=m\left(P_{i}\right)(t)+\operatorname{Post}\left(P_{i}, T_{j}\right)$.

Quando uma transição diferencial dispara, a marcação de um lugar diferencial de saída é atualizada com um novo vetor de estado contínuo. Formalmente, para $f\left(P_{i}\right)=D F$ e $f\left(T_{j}\right)=D F$, se $T_{j}$ está habilitada no tempo $t$, então $T_{j}$ dispara durante $\Delta t$, onde $\Delta t$ é o intervalo de tempo no qual $T_{j}$ permanece habilitada e $\tau$ é o passo de integração utilizado. Assim, $\forall P_{i} \in T_{j}^{\bullet}, m\left(P_{i}\right)(t+\tau)=\mathbf{X}$, onde $\mathbf{X}$ é o novo vetor de estado contínuo calculado. $\mathrm{O}$ modelo apresentado na Figura 3(a) ilustra o disparo de uma transição diferencial. A transição diferencial $T_{2}$ possui uma condição de fluxo $\dot{\mathbf{X}}$ representada pelo sistema de equações diferenciais de segunda ordem

$$
\dot{\mathbf{X}}=\left[\begin{array}{c}
\dot{x}_{1} \\
\dot{x}_{2}
\end{array}\right]=\left[\begin{array}{c}
-x_{1}-x_{2}+1 \\
x_{1}
\end{array}\right]
$$

com condição inicial igual a $m_{0}\left(P_{2}\right)(t)=\mathbf{X}_{0}=\left[\begin{array}{ll}0 & 0\end{array}\right]^{T}$. Durante o disparo de $T_{2}$, a marcação de $P_{2}$ é atualizada de acordo com a evolução contínua mostrada na Figura 3(b).

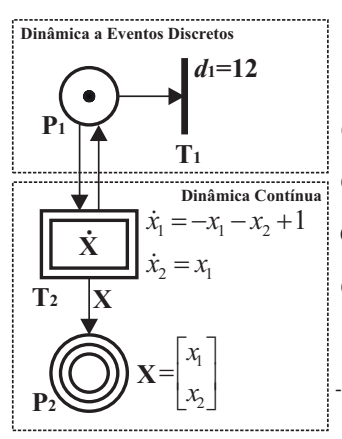

(a)

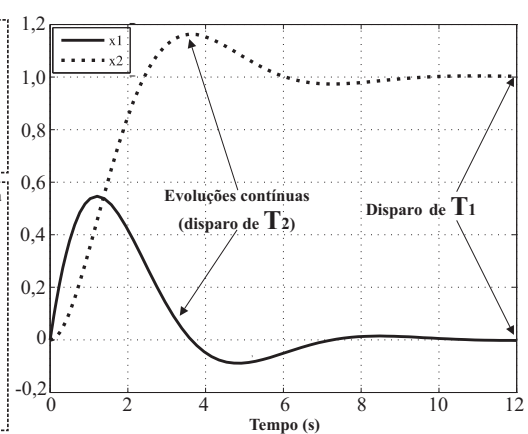

(b)
Figura 3: (a) Exemplo de modelo RPHD. (b) Evoluções contínuas resultantes do disparo de $T_{2}$.

\subsection{Modelando SHs utilizando RPHDs}

Modelar SHs é uma tarefa relativamente complexa. A concepção de modelos para esses sistemas exige do projetista uma visão clara das dinâmicas envolvidas e da forma como estas interagem. No caso das RPHDs, a modelagem de SHs, consiste das seguintes etapas:

1. Modelagem da dinâmica a eventos discretos: segue a mesma lógica utilizada para modelar Sistemas a Eventos Discretos (SEDs), ou seja, devem ser definidas as atividades e eventos discretos que caracterizam a dinâmica a eventos discretos do SH. O modelo a eventos discretos pode ser construído respeitando-se as relações de dependência entre atividades e eventos. Além disso, restrições de tempo podem ser incluídas para caracterizar as durações de atividades;

2. Modelagem da dinâmica contínua: numa RPHD, o par formado por um lugar diferencial e uma transição diferencial, é suficiente para representar uma dinâmica contínua. O cálculo da evolução contínua está associado a uma transição diferencial. Esse cálculo é realizado durante a habilitação e disparo da referida transição diferencial;

3. Modelagem da interação entre as dinâmicas: após a construção dos modelos para as duas dinâmicas, é necessário definir a forma como as mesmas interagem. Essa interação depende fundamentalmente do comportamento híbrido do sistema em questão. A interface entre as dinâmicas de um SH em uma RPHD é realizada de duas formas:

(a) Através de arcos normais (dinâmica a eventos discretos $\longrightarrow$ dinâmica contínua): esses arcos conectam lugares discretos à transições diferenciais. São responsáveis pelas condições de habilitação das mesmas e devem atender às condições definidas na Seção 3.3, ítem 1; e

(b) Através de arcos teste (dinâmica contínua $\longleftrightarrow$ dinâmica a eventos discretos): esses arcos conectam lugares diferenciais à transições discretas. Nesses arcos são definidas as funções $H_{P_{i}, T_{j}}$ e $J_{T_{j}, P_{i}}$.

Utilizando-se as RPHDs, também é possível modelar os comportamentos híbridos básicos tais como switches e jumps (também chamados de impulsos). Switches e jumps são fenômenos discretos que surgem no estudo de SHs, e podem ser autônomos ou controlados (Branicky, 1995). Em um switch, a dinâmica contínua é modificada devido a uma evolução no estado discreto. Enquanto que num jump, a dinâmica contínua é modificada sem que ocorra uma evolução 
da dinâmica a eventos discretos. Na Tabela $1^{1}$, são mostradas as conexões possíveis para o formalismo RPHDs, bem como as suas características para o processo de modelagem de SHs.

Tabela 1: Conexões possíveis para modelar SHs com as RPHDs.

\begin{tabular}{|c|c|c|c|}
\hline Conexão & Possível & Operação & Domínio \\
\hline$\underset{k=0}{\stackrel{k}{\longrightarrow}}$ & Sim & Dinâmica a eventos discretos & $k \in \mathbb{N}$ \\
\hline$\vdash \stackrel{k}{\longrightarrow}$ & Sim & Dinâmica a eventos discretos & $k \in \mathbb{N}$ \\
\hline $\mathrm{O}_{k}^{k} \underset{k}{k}$ & Sim & $\begin{array}{l}\text { Interação (dinâmica a eventos } \\
\text { discretos } \Rightarrow \text { dinâmica contínua) }\end{array}$ & $k \in \mathbb{N}$ \\
\hline (ㅇ) $\stackrel{H_{P_{i}, T_{j}}}{\longrightarrow}$ & Sim & $\begin{array}{l}\text { Interação (dinâmica contínua } \\
\Rightarrow \text { dinâmica a eventos discretos) }\end{array}$ & $H_{P_{i}, T_{j}} \in \mathbf{X}$ \\
\hline$\stackrel{J_{T_{i}, P_{i}}}{\longmapsto}$ (()) & Sim & $\begin{array}{l}\text { Interação (dinâmica a eventos } \\
\text { discretos } \Rightarrow \text { dinâmica contínua) }\end{array}$ & $J_{T_{j}, P_{i}} \in \mathbf{X}$ \\
\hline 回 ${ }^{x} \rightarrow$ (2) & Sim & Dinâmica contínua & $\mathbf{X} \subset \mathbb{R}^{l}$ \\
\hline
\end{tabular}

\section{REDES DE SENSORES SEM FIO}

As Redes de Sensores Sem Fio (RSSFs) constituem um tópico recente de pesquisa. Estas redes são compostas de dispositivos autônomos e compactos denominados nós sensores, que são equipados com vários tipos de sensores, tais como acústico, sísmico, vídeo, temperatura e pressão. Em muitas aplicações, os nós são dispostos em áreas remotas e inóspitas, o que dificulta o acesso a esses dispositivos para manutenção. Neste cenário, o tempo de vida de um nó depende diretamente da quantidade de energia disponível na sua bateria.

O hardware básico de um nó sensor (veja Figura 4) é composta de quatro subsistemas (Vieira et al., 2003): i) um $s u b$ sistema computacional que consiste de um microprocessador ou microcontrolador; ii) um subsistema de comunicação composto de um rádio de alcance curto para comunicação sem fio; iii) um subsistema de sensoriamento que liga o nó ao mundo físico e que consiste de um grupo de sensores e atuadores; e iv) um subsistema fonte de energia que alimenta o resto do nó, o qual é composto de baterias e um conversor CC-CC. O objetivo deste conversor é prover uma tensão constante para os subsistemas do nó sensor.

Uma vez que é impraticável recarregar a bateria de todos os nós, cada nó deve ser eficiente em energia. Portanto, energia é o principal recurso de um nó sensor, sendo a métrica primária para análise de uma RSSF. Para um estudo mais detalhado sobre RSSFs, o leitor deve se reportar a (Vieira et al., 2003).

\footnotetext{
${ }^{1} \mathbb{N}$ é o conjunto dos números naturais.
}

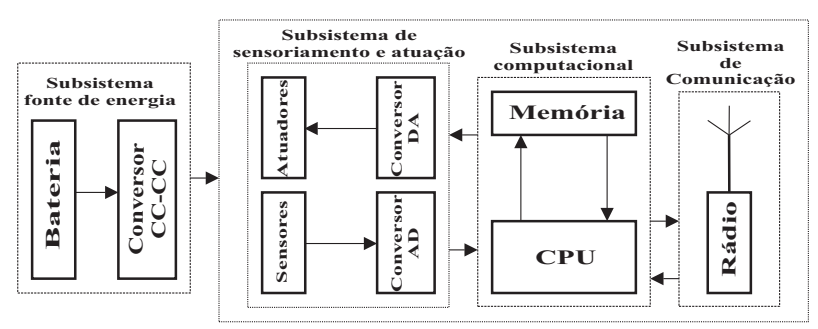

Figura 4: Hardware básico de um nó sensor.

Nas RSSFs, dinâmicas a eventos discretos e contínua coexistem e interagem, por esse motivo estas redes podem ser consideradas SHs. Sendo assim, os seus subsistemas (veja Figura 4) devem ser associados às dinâmicas inerentes a um SH. A dinâmica contínua é representada pelo subsistema fonte de energia, enquanto que a dinâmica a eventos discretos é representada pelos subsistemas de comunicação, de sensoriamento e computacional. Portanto, para estudar o consumo de energia de uma RSSF de forma mais realista é necessário levar em conta as interações entre essas dinâmicas. Neste trabalho, uma RSSF dentro do contexto dos SHs é modelada através da utilização do formalismo RPHDs. A seguir, essas dinâmicas são apresentadas.

\subsection{Dinâmica Contínua de uma RSSF}

Cada bateria do subsistema fonte de energia caracteriza-se por dois valores de tensão: (i) a tensão de circuito aberto $\left(V_{o c}\right)$ que é o valor da tensão medido nos terminais de uma bateria totalmente carregada quando não há corrente de descarga e (ii) pela tensão de corte $\left(V_{c o}\right)$ que é o valor da tensão medida nos terminais da bateria a partir do qual considera-se que a bateria está descarregada (Lahiri et al., 2002). A capacidade $C$ de uma bateria é a quantidade de energia que uma bateria entrega sob uma condição de carga, e que é normalmente usada (durante a vida da bateria) como uma métrica para julgar a eficiência da bateria. Neste artigo, $C$ é expresso em mA-ms ( $m A$ vezes $\mathrm{ms}$ ).

Dois efeitos importantes fazem com que o desempenho de uma bateria dependa do perfil de corrente de descarga. Esses efeitos são: (i) efeito de taxa de capacidade, o qual deve-se a dependência entre a capacidade atual de uma bateria e a intensidade da corrente de descarga, e (ii) efeito de recuperação, que deve-se à recuperação de carga da bateria durante períodos nos quais a corrente de descarga é reduzida significativamente. Ambos os fenômenos podem afetar a capacidade e o tempo de vida de uma bateria (Lahiri et al., 2002).

Modelos de baterias capturam as características reais de operação das mesmas, e podem ser usados para prever os seus comportamentos sob várias condições de carga/descarga. 
Modelos de baterias constituem-se em ferramentas úteis para o projeto de sistemas alimentados por baterias, porque permitem a análise do comportamento de descarga da bateria sob diferentes especificações do projeto. Modelos analíticos de baterias podem incluir modelos de carga constante e de carga variável, e conseguem capturar o efeito de taxa de capacidade e o efeito de recuperação. Esses modelos são flexíveis com respeito a implementação, e podem ser facilmente configurados para tipos de baterias específicos.

O modelo analítico mais simples é o modelo linear. Porém, as previsões do tempo de vida obtidas com este modelo não são precisas. Neste trabalho, utiliza-se o modelo analítico de (Rakhmatov and Vrudhula, 2001) para estimar o tempo de vida da bateria de um nó sensor. A seguir são apresentadas as características deste modelo analítico de bateria.

\subsubsection{Modelo Rakhmatov-Vrudhula}

Em (Rakhmatov and Vrudhula, 2001), os autores derivam equações para o cálculo do tempo de vida de uma bateria do tipo Lítio-Ion alimentando uma carga constante ou variável. Para estimar o tempo de vida da bateria, dois parâmetros específicos são necessários. O parâmetro $\alpha$ que representa a capacidade da bateria e $\beta$ que descreve o comportamento não linear da bateria durante os períodos de carga e descarga.

A equação 1 descreve o impacto do perfil de descarga no tempo de vida da bateria, onde $I_{k-1}$ é a corrente de descarga durante o período $k-1$. A função $A($.$) calcula o impacto$ do comportamento não linear da bateria, onde $L$ é o tempo de vida da bateria, $t_{k}$ é a duração do período $k$ e $t_{k-1}$ é a duração do período $k-1$. A função $A($.) e o algoritmo necessário para o cálculo de $L$ são apresentados com detalhes em (Rakhmatov and Vrudhula, 2001).

$$
\alpha=\sum_{k=1}^{n} 2 I_{k-1} A\left(L, t_{k}, t_{k-1}, \beta\right)
$$

Os parâmetros de entrada para o algoritmo de cálculo de $L$, são: (i) o conjunto com os valores de corrente de descarga $S_{I}=\left\{I_{0}, I_{1}, \ldots, I_{n}\right\}$; (ii) o conjunto com os respectivos intervalos de tempo $S_{t}=\left\{t_{0}, t_{1}, \ldots, t_{n}\right\}$; e (iii) os parâmetros $\alpha$ e $\beta$. O resultado obtido pode ser: (a) o tempo de vida da bateria, ou (b) a capacidade disponível da mesma.

Em (Handy and Timmermann, 2003) é apresentado um algoritmo para a integração do modelo analítico de bateria de Rakhmatov-Vrudhula com ambientes de simulação para redes sem fio e dispositivos móveis. Portanto, a estimação do tempo de vida de dispositivos alimentados por bateria é simplificada pois, não é necessário conhecer previamente o perfil completo de descarga da bateria. Esta abordagem é usada neste trabalho. Assim, efeitos não lineares das baterias, como efeito de taxa de capacidade e efeito de recuperação podem ser levados em conta na maximização do tempo de vida de um nó sensor.

Neste trabalho foram adotados $\alpha=2459100$ e $\beta=$ 4034. Estes parâmetros foram estimados com base nos dados de uma bateria alcalina (Panasonic, 2006) freqüentemente utilizada na alimentação de nós sensores. $\mathrm{Na}$ Figura 5 pode-se observar uma comparação entre os resultados fornecidos pelos modelos linear e RakhmatovVrudhula na previsão do consumo de energia de uma bateria. Neste caso, foi utilizado um perfil de descarga com 10 segundos de duração, o qual é formado pelos modos de operação de um nó sensor (veja Tabela 3): $114 \times$ [ $S_{I}=\{14,35,18,14,35,18,35,14,35,18,14,35,18,35\} \mathrm{mA}$ e $\left.S_{t}=\{2,8,8,2,8,8,8,2,8,8,2,8,8,8\} \mathrm{ms}\right]$. Note que o modelo linear fornece um consumo 50 vezes maior que o modelo Rakhmatov-Vrudhula. Esta grande divergência deve-se ao fato de que o modelo linear, devido a sua simplicidade, não consegue capturar o comportamento não linear das baterias.

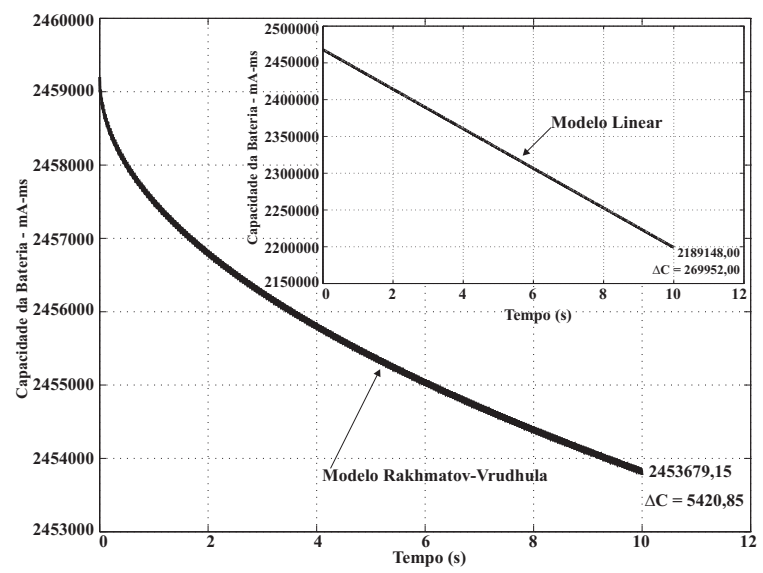

Figura 5: Capacidade de uma bateria calculada pelos modelos linear e de Rakhmatov-Vrudhula.

\subsection{Dinâmica a Eventos Discretos}

Numa RSSF, a dinâmica a eventos discretos é representada pelo hardware dos nós sensores e pelo hardware e software dos nós sensores. O software de um nó sensor é representado pelo sistema operacional e pelos protocolos de comunicação. A dinâmica a eventos discretos associada ao hardware de uma RSSF é representada pelas atividades e eventos dos subsistemas de comunicação, de sensoriamento e computacional de cada nó sensor da rede. Durante o funcionamento de um nó sensor cada estado da dinâmica a eventos discretos define a correspondente evolução da dinâmica contínua. $\mathrm{Na}$ teoria de SHs, esse comportamento é denominado um switch 
(Branicky, 1995). Assim, a medida que a dinâmica a eventos discretos evolui, o consumo de energia do nó sensor é calculado pelo modelo de bateria utilizado.

A evolução da dinâmica a eventos discretos, por sua vez, também pode ser influenciada pela dinâmica contínua. Esta influência é utilizada neste trabalho para definir quais eventos discretos devem ocorrer com o objetivo de gerenciar dinamicamente a energia do nó sensor a medida que a capacidade da bateria atinge níveis previamente determinados.

\subsection{Gerenciamento de Energia em RSSFs}

Existem várias técnicas para conservação de energia que podem ser aplicadas às RSSFs. De modo geral, estas técnicas podem ser classificadas em estáticas ou dinâmicas. As técnicas estáticas dizem respeito às características que não sofrem alterações no transcorrer da atividade da rede, por exemplo os Energy-Aware Routing Protocols. Diferentemente, as técnicas dinâmicas evoluem durante o funcionamento da rede e sua utilização possibilita uma melhora na conservação de energia, pelo nó sensor, em tempo de execução.

Técnicas de DPM (Dynamic Power Management) são técnicas dinâmicas utilizadas com o objetivo de reduzir o consumo de energia dos nós sensores e, conseqüentemente, aumentar o tempo de vida da rede. A idéia básica é desligar componentes específicos do nó sensor quando os mesmos não são utilizados, ou seja, o nó sensor deve mudar para estado de sleep após executar as atividades programadas e deve retornar ao estado active quando ocorrer um evento de interesse.

Diferentes técnicas de DPM foram propostas para reduzir o consumo de energia em sistemas que utilizam baterias, mas poucas destas consideram eventos externos para realizar essa otimização. Em (Passos et al., 2005), é proposta uma abordagem para gerenciamento de energia que utiliza o formalismo autômato híbrido e que leva em consideração alguns requisitos da aplicação para efetuar o desligamento dos nós sensores. Porém, nesta abordagem não é considerado o roteamento multi-hop pelos nós sensores nem o nível de carga da bateria na tomada de decisão para o desligamento dos nós sensores. Em (Sinha and Chandrakasan, 2001) é proposto um sistema operacional que utiliza a técnica DVS (Dynamic Voltage Scaling) para reduzir o consumo de energia do modulo processador (CPU) do nó sensor. Nesta proposta todas as tomadas de decisão ficam restritas ao nó sensor, sem nenhuma intervenção externa. Em (Luo et al., 2005), é apresentado uma adaptação do algoritmo proposto em (Sinha and Chandrakasan, 2001), onde são considerados outros fatores, tais como capacidade da bateria e área de cobertura nas trocas dos modos de operação do sensor.
Note que, em nenhum dos trabalhos citados foi explicitado o modelo de bateria utilizado. Entretanto, para desenvolver um modelo que represente de forma mais realista o ambiente de uma RSSF, em especial os ganhos obtidos utilizando técnicas de DPM, é necessário utilizar um modelo de bateria que leve em conta o seu comportamento não linear e, principalmente, o efeito de recuperação da mesma.

\subsection{Gerenciamento Dinâmico de Energia Baseado na Capacidade da Bateria}

Neste trabalho é proposta uma técnica de DPM denominada Gerenciamento Dinâmico de energia baseado na Capacidade da bateria (GDC). A medida que a capacidade da bateria diminui, atingindo níveis previamente definidos, o modo de operação do nó sensor sofre modificações. Tais modificações baseiam-se na freqüência de desligamento dos subsistemas de comunicação e sensoriamento.

O objetivo do desligamento é reduzir a corrente solicitada da bateria e com isso obter recuperação de capacidade da mesma (efeito de recuperação). Em um nó sensor, os modos de operação que, normalmente, demandam mais corrente elétrica são: transmissão (Tx) e recepção (Rx). Os ganhos de energia obtidos quando o desligamento (Intervalo de Recuperação $=2 \mathrm{~ms}$ ) é realizado após uma transmissão e após uma recepção são 58,30 e 21,57 mA-ms, respectivamente. Note que um ganho maior é obtido quando o desligamento é realizado após uma transmissão. Portanto, o desligamento dos subsistemas de comunicação e sensoriamento deve ser realizado sempre após uma transmissão. Desta forma, o efeito de recuperação torna-se mais efetivo.

Considerando que o rádio do nó sensor transmite a uma taxa de 38,4 kbps (Crossbow, 2006) e que o tamanho do pacote a ser transmitido ou recebido tem 40 bytes, a duração de uma transmissão e de uma recepção tem um valor fixo igual a 8 ms. A definição da duração de cada modo de gerenciamento foi definida com base na duração de uma transmissão.

Para definir o tempo de duração de cada modo foram utilizados cinco intervalos de recuperação. Na Figura 6 são mostrados esses intervalos e seus respectivos ganhos. Foi adotado que o tempo de duração de cada modo de gerenciamento seja igual ao intervalo $\mathrm{I}_{2}$, ou seja, $2 \mathrm{~ms}$. Outro intervalo poderia ter sido usado, porém, a escolha do intervalo $\mathrm{I}_{2}$ levou em conta o ganho de 58,30 mA-ms e um tempo de desligamento igual a $1 / 4$ do tempo de uma transmissão.

\subsubsection{Modelo RPHD da Técnica GDC}

A técnica GDC proposta foi modelada utilizando-se o formalismo RPHDs. A utilização desse formalismo possibilita investigar o comportamento de sistemas de forma automática, 


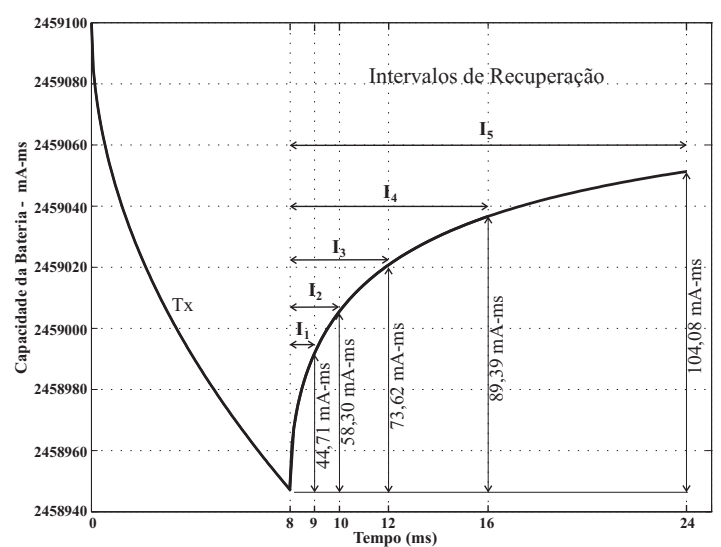

Figura 6: Cinco intervalos de recuperação $\left(I_{1} \ldots I_{5}\right)$ e seus respectivos ganhos de capacidade.

incluindo simulação e, futuramente, verificação.

Na Figura 7 é apresentado o modelo RPHD para a técnica GDC proposta. Esta técnica baseia-se na capacidade $C$ da bateria do nó sensor, representada pelo lugar diferencial $P_{1}$ $\left(m\left(P_{1}\right)(t)=\left[x_{1}\right]=C\right)$. Dependendo da capacidade da bateria, é adotado um dos três modos de gerenciamento apresentados na Tabela 2. As funções de habilitação $H_{P_{1}, T_{1}}$, $H_{P_{1}, T_{4}}$ ou $H_{P_{1}, T_{7}}$, definem a ativação dos modos GDC1 (disparo de $T_{1}$ ), GDC2 (disparo de $T_{4}$ ) ou GDC3 (disparo de $T_{7}$ ), respectivamente. A ativação de cada um destes modos é representada por uma ficha nos lugares $P_{3}, P_{6}$ ou $P_{9}$. Os modos de gerenciamento diferenciam-se pela quantidade de transmissões $\left(m\left(P_{4}\right)(t)=6, m\left(P_{7}\right)(t)=3 \mathrm{ou}\right.$ $\left.m\left(P_{10}\right)(t)=1\right)$ que devem ser realizadas antes de um desligamento. Um desligamento parcial do nó sensor (disparo de $T_{3}, T_{6}$ ou $T_{9}$ ) é representado por uma ficha no lugar $P_{11}$. O disparo da transição discreta $T_{10}$, após $d_{10}=2 \mathrm{~ms}$, reativa o nó sensor.

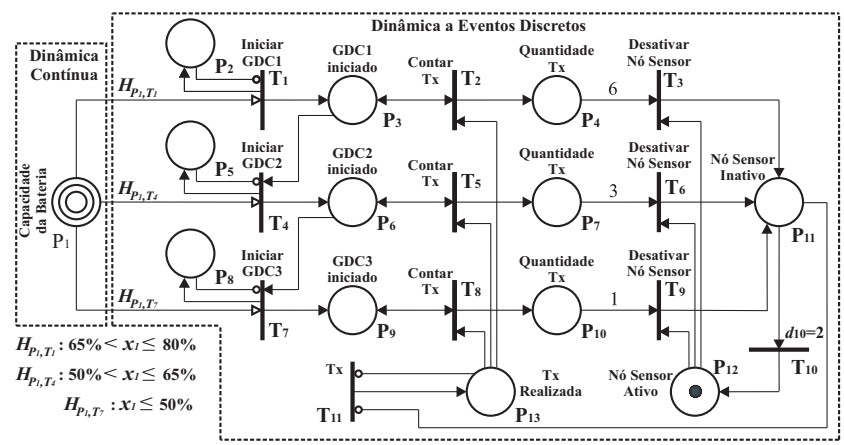

Figura 7: Modelo RPHD para a Técnica GDC proposta.

Outros modos de gerenciamento podem ser adicionados à técnica de gerenciamento dinâmico de energia proposta.
Além disso, o modelo RPHD para a técnica de gerenciamento pode ser integrado ao modelo que descreve os modos de operação de um nó sensor. Esta integração é apresentada na próxima seção.

Tabela 2: Modos de Gerenciamento Dinâmico de Energia.

\begin{tabular}{|c|c|c|}
\hline Modos & Especificação & Freqüência \\
\hline \hline GDC1 & Ativo quando: $65 \%<C \leq 80 \%$ & Após 6 transmissões \\
\hline GDC2 & Ativo quando: $50 \%<C \leq 65 \%$ & Após 3 transmissões \\
\hline GDC3 & Ativo quando: $C \leq 50 \%$ & Após 1 transmissão \\
\hline
\end{tabular}

\section{MODELO RPHD PARA UM NÓ SENSOR}

Nesta seção é apresentado o modelo RPHD para um nó sensor que utiliza a técnica de gerenciamento apresentada na Seção 4.4. O modelo RPHD para o nó sensor baseia-se em um cenário onde o nó periodicamente coleta dados do ambiente e transmite-os para um receptor. As condições de operação admitidas para o modelo são as seguintes: (a) durante os períodos de gerenciamento, o subsistema computacional passa do Modo ativo para o Modo inativo (veja a Tabela 3); (b) os subsistemas de comunicação e sensoriamento são desligados (modo Sleep) durante os períodos de gerenciamento (veja a Tabela 3); e (c) os subsistemas de comunicação e sensoriamento somente são desligados (modos GDC) quando os mesmos não estiverem executando nenhuma operação.

Os parâmetros usados neste trabalho para o nó sensor baseiam-se na especificação Berkeley Mote (veja Tabela 3), na sua versão comercial produzida e comercializada pela Crossbow Technology (Crossbow, 2006).

Tabela 3: Parâmetros do Nó Sensor.

\begin{tabular}{|c|c|}
\hline Subsistema & Especificação \\
\hline \hline Computacional & Ativo $=8 \mathrm{~mA}$, Inativo $=15 \mu \mathrm{A}$ \\
\hline Comunicação & $\mathrm{Tx}=27 \mathrm{~mA}, \mathrm{Rx}=10 \mathrm{~mA}$, Sleep $=1 \mu \mathrm{A}$ \\
\hline Sensoriamento & Ativo $=6 \mathrm{~mA}$, Sleep $=1 \mu \mathrm{A}$ \\
\hline Fonte de energia & 2 Bat.alcalina $\left(C=2459100 \mathrm{~mA}-\mathrm{ms}, V_{o c}=1,5 \mathrm{~V}, V_{c o}=0,8 \mathrm{~V}\right)$ \\
\hline
\end{tabular}

O modelo RPHD para o nó sensor é mostrado na Figura 8. Este modelo é resultado da integração, através de lugares de fusão, entre o modelo básico de um nó sensor e o modelo mostrado na Figura 7 . Neste caso, $M_{0}(t)=\left[101010000000000000 m_{0}\left(P_{19}\right)(t)\right]^{T}$, onde $m_{0}\left(P_{19}\right)(t)=\left[x_{1}(0)\right]=2459100 \mathrm{~mA}-\mathrm{ms}$.

Pode-se observar no modelo uma clara separação entre a dinâmica a eventos discretos e a contínua. A interação entre essas dinâmicas é capturada no modelo RPHD da seguinte forma. Cada estado da dinâmica a eventos discretos define a respectiva dinâmica contínua que deve evoluir enquanto o 


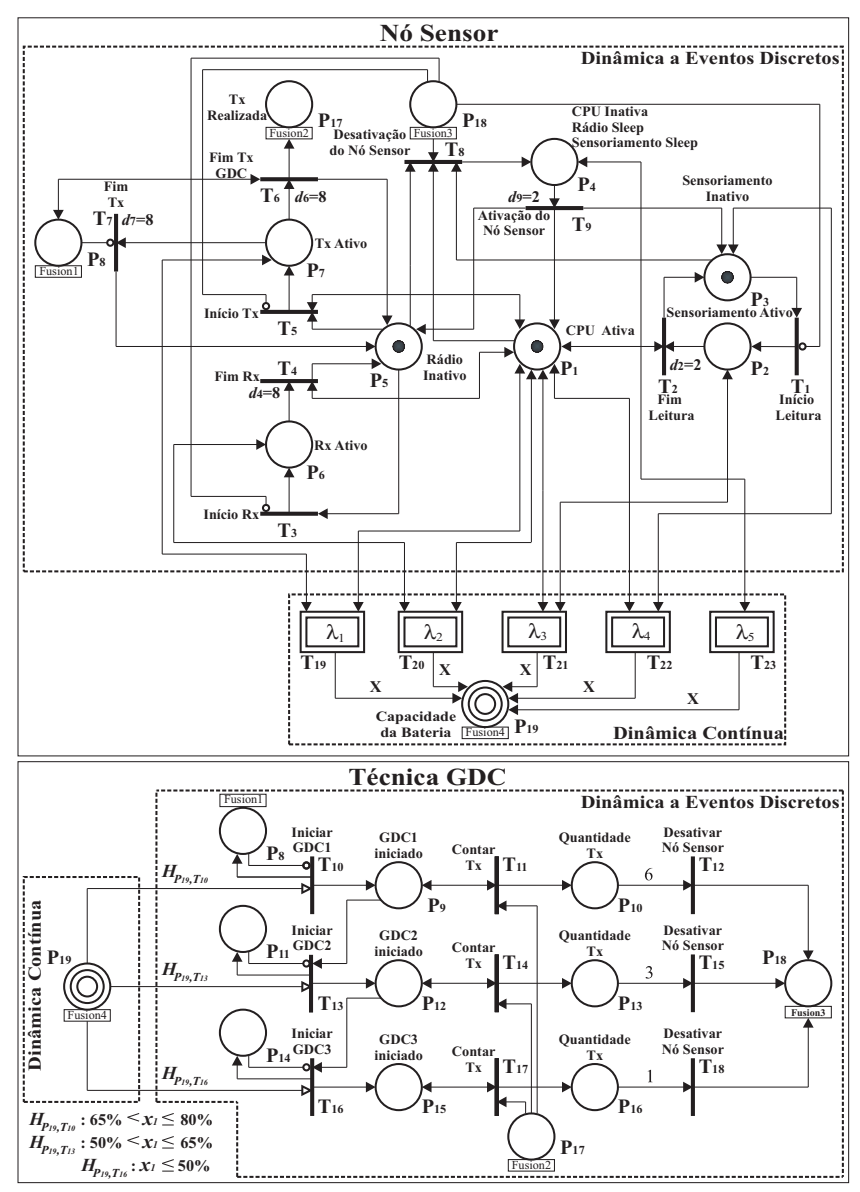

Figura 8: Modelo RPHD para um nó sensor que utiliza a técnica GDC apresentada na Seção 4.4.

sistema estiver naquele estado. Essa definição dá-se através da habilitação das transições diferenciais. Uma vez habilitada, cada transição diferencial ativa a sua respectiva função $\lambda_{i}$. Cada função $\lambda_{i}(i=1 \ldots 5)$, quando ativada executa o seguinte: (i) insere um valor de corrente $I_{k}$ e um valor de tempo $t_{k}$ nos conjuntos $S_{I}$ e $S_{t}$, respectivamente; e (ii) ativa a execução do algoritmo iterativo de cálculo do tempo de vida da bateria para o novo perfil de descarga. Portanto, com a evolução da dinâmica a eventos discretos, o perfil de descarga é atualizado e a capacidade atual da bateria é calculada.

Na fase inicial de funcionamento do nó sensor, considerando que a bateria está com sua capacidade máxima, o nó sensor pode trabalhar com todos os seus subsistemas ativos sem nenhuma restrição de operação, ou seja, sem emprego de nenhum modo de gerenciamento. Entretanto, quando a capacidade da bateria atinge um valor menor ou igual $80 \%$ e maior que $65 \%$, a transição $T_{10}$ é habilitada e o seu disparo coloca uma ficha no lugar $P_{9}$ habilitando o modo GDC1. Este mesmo procedimento repete-se quando a capacidade da ba- teria atinge $65 \%$ e $50 \%$, habilitando as transições $T_{13}$ e $T_{16}$, respectivamente.

\subsection{Operação: Nó Sensor + Técnica GDC}

Para demonstrar a utilização da técnica GDC proposta, é admitida a seguinte marcação inicial $M_{0}(t)=$ [1010100115000000001966010 $]^{T}$ para o modelo RPHD mostrado na Figura 8. Esta marcação representa o estado em que o nó sensor opera no modo GDC1 e 5 transmissões foram realizadas, ou seja, $m\left(P_{10}\right)(t)=5$. Dentre as várias sequiências de eventos que podem ser geradas a partir desta marcação, foi considerada a seguinte: $T_{1} T_{21} T_{2} T_{5} T_{19} T_{6} T_{11} T_{12} T_{8} T_{23} T_{9}$. Esta seqüência de eventos resulta na mudança do modo de operação do nó sensor para o modo inativo e na habilitação da transição diferencial $T_{23}$. Esta transição é responsável pelo cálculo da capacidade da bateria enquanto o nó sensor permanecer neste modo. $\mathrm{O}$ sistema permanece neste estado por $2 \mathrm{~ms}$. A seguir, com o disparo de $T_{9}$ o nó sensor é reativado. Na Figura 9 são mostradas as evoluções das dinâmicas associadas a esta sequiência de eventos.

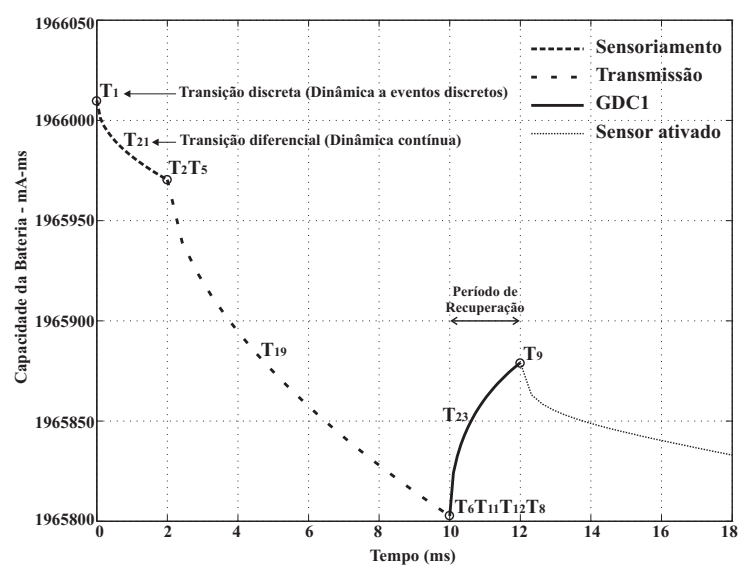

Figura 9: Exemplo de evoluções das dinâmicas do modelo RPHD mostrado na Figura 8.

Note que o modelo RPHD da técnica GDC apresentado na Seção 4.4.1 pode ser visto como um supervisor híbrido. A sua integração ao modelo RPHD do nó sensor (veja a Figura 8) permite que as especificações da Tabela 2 sejam atendidas. O supervisor híbrido atua observando o comportamento das duas dinâmicas e decide quais eventos discretos e dinâmicas contínuas podem ocorrer num determinado estado. Esta característica, pode ser vista no exemplo, da seguinte forma. A ativação de um modo GDC não depende apenas da capacidade da bateria (dinâmica contínua), depende também da quantidade de transmissões que são realizadas (dinâmica a eventos discretos). 
A síntese do supervisor híbrido mostrado na Figura 7 não foi realizada de forma automática, entretanto, as especificações desejadas foram atendidas. Outras especificações para o comportamento de um nó sensor também podem ser definidas. Para isso, os conceitos apresentados em (Koutsoukos et al., 2000; Antsaklis and Koutsoukos, 2002) podem ser utilizados para garantir a síntese automática de supervisores híbridos.

\section{RESULTADOS DAS SIMULAÇÕES}

Nesta seção são apresentados os resultados das simulações realizadas com os diferentes modos de gerenciamento. Para simular a implementação destes modos, descritos na Seção 5, foi gerado um perfil de descarga a partir do espaço de estado discreto do modelo RPHD. O perfil de descarga utilizado representa as operações básicas executadas por um nó sensor desconsiderando os períodos de desligamento dos subsistemas. Para cada simulação foram consideradas 500.000 operações realizadas pelo nó sensor. Basicamente, os modos de gerenciamento são comparados em termos de recuperação da capacidade da bateria, uma vez que a capacidade consumida durante a execução de cada ciclo é igual. Além disso, não foi considerado o consumo de energia durante o desligamento/religamento dos subsistemas do nó sensor.

Na Tabela 4 são apresentados os resultados das simulações do consumo de energia de um nó sensor com e sem adoção da técnica de gerenciamento. Quando comparam-se os ganhos de energia obtidos, verifica-se que o modo GDC3 apresenta o maior ganho. Isso acontece devido a freqüência de desligamento dos subsistemas (de acordo com a Tabela 2). Verificou-se que quanto maior a frequiência de desligamento maior é o ganho de capacidade.

Tabela 4: Resultados de simulação - Ganhos de Capacidade.

\begin{tabular}{|c|c|c|c|}
\hline Modos de Operação & $C_{\text {inicial }}(\mathrm{mA}-\mathrm{ms})$ & $C_{\text {final }}(\mathrm{mA}-\mathrm{ms})$ & Ganho (\%) \\
\hline \hline Sem GDC & 1967280,00 & 1761035,42 & \multirow{2}{*}{1,15} \\
\hline GDC1 (Sleep = 2 ms) & 1967280,00 & 1781332,83 & \multirow{2}{*}{2,34} \\
\hline \hline Sem GDC & 1598415,00 & 1392170,42 & \multirow{2}{*}{5,60} \\
\hline \hline GDC2 (Sleep = 2 ms) & 1598415,00 & 1424758,86 & \\
\hline \hline Sem GDC & 1229550,00 & 1023305,42 & \multirow{2}{|c}{} \\
\cline { 1 - 3 } GDC3 (Sleep = 2 ms) & 1229550,00 & 1080625,53 & \\
\cline { 1 - 3 }
\end{tabular}

A utilização do modelo de Rakhmatov-Vrudhula neste trabalho, apresentou a vantagem de capturar o comportamento não linear de uma bateria, permitindo um estudo mais realista das RSSFs. Portanto, foi possível verificar a eficácia da técnica GDC proposta.

Entretanto, o ganho em qualidade exige um elevado custo computacional para simular a descarga completa de uma bateria. Assim, neste trabalho, ao invés de buscar o resul- tado final para o ganho no tempo de vida do nó sensor com a aplicação da técnica GDC proposta, optou-se por utilizar os resultados parciais mostrados na Tabela 4 para demonstrar que esse ganho existe e é considerável. A busca por um desempenho computacional melhor do modelo de Rakhmatov-Vrudhula é necessaria e deverá ser realizada para permitir a sua utilização num cenário mais realista de uma RSSF.

\section{CONCLUSÕES}

Neste trabalho, foi apresentado um formalismo denominado Redes de Petri Híbridas Diferenciais. Este formalismo tem o propósito de modelar e simular SHs. Ele baseia-se nos principais elementos da semântica do autômato híbrido e no poder de modelagem das redes de Petri.

Entretanto, o formalismo RPHDs, em sua versão atual, apresenta a dificuldade para modelar SHs que têm uma estrutura de dados discreta muito complexa. Esta mesma dificuldade existe em (LeBail et al., 1991; Alla and David, 2004), por exemplo. Isto deve-se a utilização de um tipo simples de ficha (ficha preta), o que acarreta uma estrutura a eventos discretos maior, ou seja, uma quantidade maior de lugares discretos com diversos predicados. Contudo, o formalismo RPHDs tem se mostrado bastante eficiente na modelagem e simulação de SHs, e suas versões futuras deverão contemplar a solução para este problema.

Considerando que RSSFs são SHs, o formalismo RPHDs foi aplicado neste trabalho na modelagem de uma técnica proposta para o gerenciamento dinâmico de energia de um nó sensor. Portanto, ao levar em conta as interações entre as dinâmicas destas redes obtém-se resultados mais realistas no estudo do consumo de energia. Esta técnica é denominada Gerenciamento Dinâmico de energia baseado na Capacidade da bateria (GDC), a qual baseia-se na capacidade da bateria e no desligamento dos subsistemas do nó sensor. Assim, ao invés de utilizar uma estratégia de desligamento por um longo período de tempo, optou-se por distribuir o mesmo durante os ciclos de trabalho do nó sensor.

A utilização da técnica GDC associada a um modelo preciso de bateria permitiu que efeitos não lineares das baterias, como efeito de taxa de capacidade e efeito de recuperação pudessem ser levados em conta na previsão do tempo de vida de um nó sensor. Note que a adoção da referida técnica produziu uma recuperação de capacidade da bateria que alimenta o nó sensor e, não apenas, uma economia devido o desligamento parcial do nó sensor.

Como trabalhos futuros, existe a necessidade de dar prosseguimento ao desenvolvimento do formalismo RPHDs para estudar SHs. Para analisar formalmente SHs, pretende-se: 
(i) utilizar análise de invariantes (LeBail et al., 1991; Alla and David, 2004), e (ii) integrar ao formalismo os métodos de verificação formal normalmente utilizados nos autômatos híbridos. Desta forma, propriedades como segurança (safety properties), vivacidade (liveness properties) e restrições de tempo (timeliness properties) poderão ser analisadas.

Pretende-se também, realizar a integração entre a técnica GDC proposta com os requisitos da aplicação no ambiente de uma RSSF. Tal integração deverá ser útil na tomada de decisão de qual modo de gerenciamento deverá ser utilizado pelo nó sensor. Além disso, outras técnicas de gerenciamento de energia podem ser aplicadas a uma RSSF através da aplicação dos conceitos de Controle Supervisório Híbrido (Antsaklis and Koutsoukos, 2002).

\section{AGRADECIMENTOS}

Os autores agradecem à CAPES e o CNPq pelo apoio financeiro para a realização desse trabalho.

\section{REFERÊNCIAS}

Alla, H. and David, R. (2004). Discrete, Continuous, and Hybrid Petri Nets, Springer-Verlag.

Antsaklis, P. J. and Koutsoukos, X. D. (2002). Hybrid Systems Control, Encyclopedia of Physical Science and Technology, Third Edition, 7: 445-458.

Branicky, M. S. (1995). Studies in Hybrid Systems: Modeling, Analysis, and Control, PhD thesis, MIT, Cambridge, USA.

Champagnat, R. (1998). Supervision des Systèmes Discontinus: Definition d'un Modèle Hybride et Pilotage en Tempsrèel, PhD thesis, Universitè Paul Sabatier, Toulouse, France.

Crossbow (2006). Crossbow technology inc. data sheet: http://www.xbow.com.

Dang, T. (2000). Verification and Synthesis of Hybrid Systems, PhD thesis, Institut National Polytechnique de Grenoble - VERIMAG.

Daubas, B., Pagès, A. and Pingaud, H. (1994). Combined simulation of hybrid processes, IEEE-SMC, pp. 320325 .

David, R. and Alla, H. (1987). Continuous petri nets, 8th European Workshop on Applications and Theory of Petri Nets, Zaragoza, pp. 274-294.

Demongodin, I. and Koussoulas, N. T. (1996). Modeling dynamic systems through petri nets, CESA, pp. 279284.
Drath, R. (1998). Hybrid object nets: An object oriented concept for modelling complex hybrid systems, ADPM, Reims, pp. 436-442.

Dubois, E., Alla, H. and David, R. (1994). Continuous petri nets with maximal speeds depending on time, Proc. $15^{\text {th }}$ ICATPN, Zaragoza, Spain, pp. 32-39.

Giua, A. and Usai, E. (1998). Modeling hybrid systems by high-level petri nets, European Journal of Automation APII-JESA, 32(9-10/98): 1209-1231.

Handy, M. and Timmermann, D. (2003). Simulation of mobile wireless networks with accurate modelling of nonlinear battery effects, IASTED-ASM, pp. 532-537.

Koutsoukos, X. D., Antsaklis, P. J., Stiver, J. A. and Lemmon, M. D. (2000). Supervisory Control of Hybrid Systems, IEEE, Special Issue on Hybrid Systems, 88(7): 1026-1049.

Lahiri, K., Raghunathan, A., Dey, S. and Panigrahi, D. (2002). Battery-driven system design: a new frontier in low power design, IEEE Comp. Society - VLSID, pp. 17.

LeBail, J., Alla, H. and David, R. (1991). Hybrid petri nets, Proc. 1st ECC, Grenoble, France, pp. 187-191.

Luo, R. C., Tu, L. C. and Chen, O. (2005). An efficient dynamic power management policy on sensor network, Proceedings of AINA, IEEE Computer Society, pp. 341344.

Murata, T. (1989). Petri nets: Properties, analysis and applications, Proc. of IEEE, 77(4): 541-580.

Panasonic (2006). Panasonic industrial alkaline batteries data sheet: http://www.panasonic.com.

Passos, R. M., Coelho Jr., C. J. N., Loureiro, A. A. F. and Mini, R. A. F. (2005). Dynamic power management in wireless sensor networks: An application-driven approach, WONS-IEEE Computer Society, pp. 109-118.

Rakhmatov, D. and Vrudhula, S. (2001). An analytical highlevel battery model for use in energy management of portable electronic systems, Proc. of ICCAD, pp. 1-6.

Rezai, M., Ito, M. R. and Lawrence, P. D. (1995). Modeling and simulation of hybrid control systems by global petri nets, IEEE-ISCAS, pp. 908-911.

Sinha, A. and Chandrakasan, A. (2001). Dynamic power management in wireless sensor networks, IEEE Design Test of Computer, pp. 62-74. 
Sousa, J. R. B., Lima, A. M. N. and Perkusich, A. (2005). Redes de Petri Híbridas Diferenciais: Aplicação na Modelagem do Consumo de Energia de um Nó Sensor Sem Fio, SBAI, São Luis, MA, Brasil, pp. 1-8.

Vieira, M. A. M., Coelho Jr., C. N., da Silva Jr., D. C. and da Mata, J. M. (2003). Survey on wireless sensor network devices, IEEE-ETFA, 1(16-19): 537-544.

Villani, E. (2004). Modelagem e Análise de Sistemas Supervisórios Híbridos, $\mathrm{PhD}$ thesis, Esc. Politécnica da USP.

Wu, Y., Wu, W., Zeng, J., Sun, G., Su, H. and Chu, J. (2002). Modeling and simulation of hybrid dynamical systems with generalized differential petri nets, IEEEISIC, Vancouver, Canada, pp. 789-794. 